\title{
BINDING ENERGY OF A BOUND POLARON IN A QUANTUM WELL WIRE
}

\author{
M. Bouhassoune ${ }^{a}$, R. Charrour ${ }^{a}$, M. Fliyou $^{b}$, D. Bria ${ }^{a}$ \\ AND A. NOUGAOUI ${ }^{a}$ \\ ${ }^{a}$ Laboratoire de Dynamique et d'Optique des Matériaux, Faculté des Sciences \\ Université Mohamed ler, Oujda, Morocco \\ ${ }^{b}$ Equipe de Physique du Solide, ENS, B.P. 5206 Benssouda, Fès, Morocco
}

(Received April 11, 2000)

Theoretical study of the binding energies of an off-center donor hydrogenic impurity in a cylindrical quantum well wires semiconductor is presented. Calculations are performed in the framework of the effective mass approximation using the variational approach. We describe the effect of the quantum confinement by an infinitely deep potential well and we take into consideration the interaction between the charge carrier (electron and ion) and the optical phonons (confined longitudinal optical and surface optical). Our results show that the impurity binding energy depends strongly on the spatial confinement, the impurity position and the polaronic corrections.

PACS numbers: 71.38.+i, 73.20.Dx, 73.20.Hb, 73.20.Fz

\section{Introduction}

With recent progress in nanofabrication technology, it has been possible to fabricate new structures of semiconductor such as two-dimensional quantum wells (QWs), one-dimensional quantum well wires (QWWs) and zero-dimensional quantum dots (QDs). Extensive theoretical and experimental work have been devoted to the study of the optical and electronic properties presented by these nanostructures because of their possible application in optoelectronic device [1-15].

The presence of the impurities in these systems contributes to additional response when external probes are applied. Since the pioneer work realized by Bastard [1] of the hydrogenic impurity binding energy in quantum well, extensive investigation has been reported as a function of different parameters such as the spatial confinement, the impurity position and the applied external field (electric and magnetic field) [16-22].

Since the carrier-charge phonon interactions are essential to understand the experimental observation spectrum in semiconductor [23], the polaronic effect has 
become a main problem in the physics of low-dimensional systems. Recently various investigations, on electronic properties in low-dimensional semiconductor, have taken into consideration the effect of the electron-phonon interaction. Zhou and $\mathrm{Gu}[24]$ have studied the cyclotron resonance of the electron in cylindrical quantum well wire by taking into account the interaction of the electron with the surface optical (SO) phonon modes. They have shown that the polaron self-energy and the cyclotron frequencies decrease as the wire size increases. Sheng et al. [25] have investigated the polaron self-energy and the renormalized effective mass due to the SO phonon modes. Zheng and Matsuura [26] have studied the effect of both the confined longitudinal optical (LO) and interface optical (IO) phonons on the exciton binding energy. Chen et al. [27] have investigated the thickness dependence of the binding energy of an impurity bound polaron in a parabolic quantum dot subject to a magnetic field by using the second-order perturbation theory and taking into consideration the electron-bulk LO phonon coupling. They have shown that the effect of the electron-phonon interaction enhances the binding energy. In the previous theoretical investigation, the ion-phonon interaction is not included. This last interaction affects the polaronic contribution to the binding energy as it was pointed out by Marini et al. [28] for a donor-like exciton in spherical quantum dots and Sheng and Gu [29] for the binding energy for exciton in cylindrical QWW.

In this work, we report a variational calculation of an off-center donor impurity binding energy in cylindrical quantum well wire embedded in dielectric matrix, within the effective mass approximation. We consider that the electrons are confined in an infinitely deep potential well. The coupling effect of different phonon modes (confined LO and SO) with the charge carrier (electron and ion) is included. In Sec. 2 we describe the theoretical model based on a variational approach, in $\mathrm{Sec} .3 \mathrm{we}$ give the numerical results and discussion. The conclusion is given in the last section.

\section{Theoretical model}

The Hamiltonian of a shallow hydrogenic impurity in a cylindrical quantum well wire, embedded in dielectric matrix, interacting with different optical phonon modes (confined LO and $\mathrm{SO}$ ), is written as

$$
H=H_{\mathrm{e}}+H_{\mathrm{ph}}+H_{\mathrm{e}-\mathrm{LO}}+H_{\mathrm{e}-\mathrm{SO}}+H_{\text {ion-LO }}+H_{\text {ion-so }} .
$$

$H_{\mathrm{e}}$ is the electronic Hamiltonian given by

$$
H_{\mathrm{e}}=\frac{P^{2}}{2 m^{*}}-\frac{e^{2}}{\varepsilon_{\infty} \sqrt{\left(\rho-\rho_{0}\right)^{2}+z^{2}}}+V_{\mathrm{c}}(r)
$$

where $m^{*}$ is the electron effective mass and $\rho_{0}$ is the impurity position. The $z$ coordinate gives the relative separation of the electron from the impurity ion along the axis of the quantum well wire. In the absence of the phonon modes, the Coulomb potential will be taken as $\frac{-e^{2}}{\varepsilon_{0} \sqrt{\left(\rho-\rho_{0}\right)^{2}+z^{2}}} \cdot \varepsilon_{0}$ and $\varepsilon_{\infty}$ are, respectively, the low and high dielectric constant. $V_{\mathrm{c}}(r)$ is the confining potential taken as

$$
V_{c}(r)=\left\{\begin{array}{cc}
0 & \text { if } \rho \leq R \\
\infty & \text { elsewhere }
\end{array}\right.
$$


The second term in (1) is the total Hamiltonian of the free phonon field in cylindrical QWW

$$
\begin{aligned}
& H_{\mathrm{ph}}=H_{\mathrm{LO}}+H_{\mathrm{SO}}, \\
& H_{\mathrm{LO}}=\sum_{m, n, k} \hbar \omega_{\mathrm{LO}} A_{m n}^{+}(k) A_{m m}(k), \\
& H_{\mathrm{SO}}=\sum_{m, k} \hbar \omega_{\mathrm{SO}} B_{m}^{+}(k) B_{m}(k),
\end{aligned}
$$

where $A_{m n}^{+}(k)\left[A_{m n}(k)\right]$ is the creation [annihilation] operator of the confined longitudinal optical phonons with the frequency $\omega_{\text {LO }}$ (they suit the commutative rules of bosons) and the wave vector $\left(k_{\|}=\chi_{m n} / R, k\right)$, where $\chi_{m n}$ is the $n$-th root of the $m$-th order Bessel function and $k$ is the projection of the wave vector on the $z$-axis of the wire. $B_{m}^{+}(k)$ and $B_{m}(k)$ are the operators corresponding to the surface optical phonons with the frequency $\omega_{\mathrm{So}}$.

The eigenfrequencies for different modes of phonon are obtained by using the standard boundary conditions of electrostatic and taking into consideration the geometry of the cylindrical QWW [30]

$$
\begin{aligned}
& \omega_{\mathrm{LO}}^{2}=\frac{\varepsilon_{0}}{\varepsilon_{\infty}} \omega_{\text {TO }}^{2}, \\
& \omega_{\text {SO }}^{2}=\frac{\varepsilon_{0}-\varepsilon\left(\omega_{\mathrm{SO}}\right)}{\varepsilon_{\infty}-\varepsilon\left(\omega_{\mathrm{SO}}\right)} \omega_{\text {TO }}^{2},
\end{aligned}
$$

where

$$
\varepsilon\left(\omega_{\mathrm{SO}}\right)=\varepsilon_{\mathrm{d}} \frac{I_{m}(k R) K_{m}^{\prime}(k R)}{K_{m}(k R) I_{m}^{\prime}(k R)},
$$

$\omega_{\text {TO }}$ is the transverse optical phonon frequency and $\varepsilon_{\mathrm{d}}$ is the matrix constant dielectric. $I_{m}, K_{m}$ are the modified Bessel functions and $I_{m}^{\prime}, K_{m}^{\prime}$ are the first derivatives of the modified Bessel functions of $m$-th order with argument $k R$.

$$
\begin{aligned}
& H_{\mathrm{e}-\mathrm{LO}}=-\sum_{m, n, k}\left\{\left[V_{m n} J_{m}\left(\chi_{m n} \rho / R\right) \mathrm{e}^{\mathrm{i} m \varphi} \mathrm{e}^{\mathrm{i} k z_{\mathrm{e}}} A_{m n}(k)\right]+\text { c.c. }\right\}, \\
& H_{\text {ion-LO }}=-\sum_{m, n, k}\left\{\left[V_{m n} J_{m}\left(\chi_{m n} \rho_{0} / R\right) \mathrm{e}^{\mathrm{i} m \varphi_{0}} \mathrm{e}^{\mathrm{i} k z_{0}} A_{m n}(k)\right]+\text { c.c. }\right\} .
\end{aligned}
$$

$H_{\mathrm{e}-\mathrm{LO}}$ and $H_{\text {ion-LO }}$ describe the interaction Hamiltonian of an electron and an ion with the confined LO phonon modes, respectively [31]. $V_{m n}$ is their corresponding Fourier coefficient given by

$$
V_{m n}^{2}=\frac{1}{V_{0}} \frac{2 \pi \mathrm{e}^{2} \hbar \omega_{\mathrm{LO}}}{J_{m+1}^{2}\left(\chi_{m n}\right)\left(\chi_{m n}^{2} / R^{2}+k^{2}\right)}\left(\frac{1}{\varepsilon_{\infty}}-\frac{1}{\varepsilon_{0}}\right),
$$

where $V_{0}=\pi R^{2} L_{z}$ is the crystal volume and $L_{z}$ is the length of the wire. $H_{\mathrm{e}-\mathrm{LO}}$ and $H_{\text {ion-Lo }}$ are the Hamiltonian interaction of an electron and an ion with the SO phonon modes

$$
H_{\mathrm{e}-\mathrm{SO}}=-\sum_{m, k}\left\{\left[\Gamma_{m} I_{m}(k \rho) \mathrm{e}^{\mathrm{i} m \varphi} \mathrm{e}^{\mathrm{i} k z_{e}} B_{m}(k)\right]++ \text { c.c. }\right\},
$$




$$
H_{\text {ion-SO }}=-\sum_{m, k}\left\{\left[\Gamma_{m} I_{m}\left(k \rho_{0}\right) \mathrm{e}^{\mathrm{i} m \varphi_{0}} \mathrm{e}^{\mathrm{i} k z_{0}} B_{m}(k)\right]+\text { c.c. }\right\},
$$

and

$$
\Gamma_{m}^{2}=\frac{\mathrm{e}^{2} \hbar \omega_{\mathrm{SO}}}{L_{z} k R I_{m}(k R) I_{m}^{\prime}(k R)}\left[\frac{1}{\varepsilon\left(\omega_{\mathrm{SO}}\right)-\varepsilon_{0}}-\frac{1}{\varepsilon\left(\omega_{S O}\right)-\varepsilon_{\infty}}\right] .
$$

In the following, we shall adopt the variational treatment developed by Lee et al. [32], for quasi-one-dimensional systems, to deal with the Hamiltonian (1). We use the following unitary transformations:

$$
U_{1}=\exp \left\{-\frac{\mathrm{i}}{\hbar} z\left[\sum_{m, n, k} \hbar k A_{m n}^{+}(k) A_{m n}(k)+\sum_{m, k} \hbar k B_{m}^{+}(k) B_{m}(k)\right]\right\},
$$

and

$$
U_{2}=\exp \left\{\sum_{m, n, k}\left[f_{m n}(k) A_{m n}^{+}(k)-\text { c.c. }\right]+\sum_{m, k}\left[g_{m}(k) B_{m}^{+}(k)-c . c .\right]\right\},
$$

where $f_{m n}(k)$ and $g_{m}(k)$ are the functions, which are determined by the variational means.

For the surface phonons, the inter-subband transition can be neglected and the electrons are assumed to occupy only the lowest-subband state $(n=1$, $m=0)$. We only consider the lowest surface phonon mode $(m=0)[25]$.

At low temperature limit, few phonons are excited, we can consider that $|0,0\rangle$ is the ground state of phonons. The wave function of the system can be written as

$$
|\psi(\rho, \varphi, z)\rangle=\left|\phi_{\mathrm{e}}(\rho, \varphi, z)\right\rangle|0,0\rangle,
$$

where $\phi_{\mathrm{e}}(\rho, \varphi, z)$ is the electron wave function in cylindrical quantum well wire.

The expectation value of the Hamiltonian $H^{*}$ in such a state is given by

$$
E=\left\langle\psi(\rho, \varphi, z)\left|H^{*}\right| \psi(\rho, \varphi, z)\right\rangle=\left\langle\phi_{\mathrm{e}}(\rho, \varphi, z)\left|H_{\mathrm{p}}\right| \phi_{\mathrm{e}}(\rho, \varphi, z)\right\rangle,
$$

where we set

$$
H_{\mathrm{p}}=\left\langle 0,0\left|H^{*}\right| 0,0\right\rangle \text {. }
$$

From the variational conditions $\frac{\partial H_{\mathrm{p}}}{\partial f_{n}^{*}}=\frac{\partial H_{\mathrm{p}}}{\partial g^{*}}=\frac{\partial H_{\mathrm{p}}}{\partial f_{n}}=\frac{\partial H_{\mathrm{p}}}{\partial g}=0$ we can obtain

$$
\begin{aligned}
& f_{n}(k)=\frac{V_{0 n}\left[J_{0}\left(\chi_{0 n} \rho / R\right)-J_{0}\left(\chi_{0 n} \rho_{0} / R\right) \mathrm{e}^{-\mathrm{i} k\left(z_{\mathrm{e}}-z_{0}\right)}\right]}{\hbar \omega_{\mathrm{LO}}+\frac{\hbar^{2} k^{2}}{2 m^{*}}-\left(1-\eta_{1}\right) \frac{\hbar^{2} k}{m^{*}} q_{z}}, \\
& g(k)=\frac{\Gamma_{0}\left[I_{0}\left(k_{z} \rho\right)-I_{0}\left(k_{z} \rho_{0}\right) \mathrm{e}^{-\mathrm{i} k\left(z_{\mathrm{e}}-z_{0}\right)}\right]}{\hbar \omega_{\mathrm{SO}}+\frac{\hbar^{2} k^{2}}{2 m^{*}}-\left(1-\eta_{2}\right) \frac{\hbar^{2} k}{m^{*}} q_{z}},
\end{aligned}
$$

$f_{n}^{*}$ and $g^{*}$ are simply the conjugate formulas of Eqs. (21) and (22).

In the above expressions, we have introduced the parameters $\eta_{1}$ and $\eta_{2}$ such as

$$
\sum_{n, k} k\left|f_{n}(k)\right|^{2}=\eta_{1} q_{z}
$$




$$
\sum_{k} k|g(k)|^{2}=\eta_{2} q_{z}
$$

By putting formulas (21), (22) and their conjugates into (23), (24) respectively and expanding them to the first power of $q_{z}$ (electron wave vector), $\eta_{1}$ and $\eta_{2}$ will be given by

$$
\begin{aligned}
& \eta_{1}=\frac{\alpha C}{1+\alpha C} \\
& \eta_{2}=\frac{\alpha D}{1+\alpha D}
\end{aligned}
$$

where

$$
\begin{gathered}
C=\frac{16 R^{3} k_{\mathrm{LO}}^{3}}{\pi} \sum_{n} \frac{1}{J_{1}^{2}\left(\chi_{0 n}\right)} \\
\times \int_{0}^{\infty} \frac{y^{2}\left[J_{0}^{2}\left(\chi_{0 n} \frac{\rho}{R}\right)+J_{0}^{2}\left(\chi_{0 n} \frac{\rho_{0}}{R}\right)-2 \cos \left(y \frac{z_{e}-z_{0}}{R}\right) J_{0}\left(\chi_{0 n} \frac{\rho}{R}\right) J_{0}\left(\chi_{0 n} \frac{\rho_{0}}{R}\right)\right]}{\left(\chi_{0 n}^{2}+y^{2}\right)\left[\left(R k_{\mathrm{LO}}\right)^{2}+y^{2}\right]^{3}} \mathrm{~d} y
\end{gathered}
$$

and

$$
\begin{gathered}
D=\frac{8 R^{3} k_{\mathrm{LO}}^{3} \varepsilon_{\infty}^{3 / 2} \varepsilon_{0}^{1 / 2}}{\pi} \\
\times \int_{0}^{+\infty} \frac{y \Omega_{0}(y)\left[I_{0}^{2}\left(y \frac{\rho}{R}\right)+I_{0}^{2}\left(y \frac{\rho_{0}}{R}\right)-2 \cos \left(y \frac{z_{e}-z_{0}}{R}\right) I_{0}\left(y \frac{\rho_{0}}{R}\right)\right]}{I_{0}(y) I_{1}(y)\left[\varepsilon\left(\omega_{\mathrm{SO}}\right)-\varepsilon_{0}\right]\left[\varepsilon\left(\omega_{S O}\right)-\varepsilon_{\infty}\right]\left[\left(\frac{\varepsilon_{\infty}}{\varepsilon_{0}}\right)^{1 / 2} \Omega_{0}(y) R^{2} k_{\mathrm{LO}}^{2}+y^{2}\right]^{3}} \mathrm{~d} y
\end{gathered}
$$

with

$$
\Omega_{0}(y)=\left[\frac{\varepsilon_{0} K_{0}(y) I_{1}(y)+I_{0}(y) K_{1}(y)}{\varepsilon_{\infty} K_{0}(y) I_{1}(y)+I_{0}(y) K_{1}(y)}\right]^{1 / 2},
$$

$k_{\mathrm{LO}}=\sqrt{2 m^{*} \omega_{\mathrm{LO}} / \hbar}$ is the polaron wave vector and $\alpha=\frac{m^{*} e^{2}}{\hbar^{2} k_{\mathrm{LO}}^{2}}\left(\frac{1}{\varepsilon_{\infty}}-\frac{1}{\varepsilon_{0}}\right)$ is the coupling constant of the electron-LO phonon interaction.

The effective Hamiltonian $H_{\mathrm{p}}$ becomes

$$
\begin{aligned}
H_{\mathrm{p}}= & -\frac{\hbar^{2}}{2 m^{*}}\left(\frac{\partial^{2}}{\partial \rho^{2}}+\frac{1}{\rho} \frac{\partial}{\partial \rho}+\frac{1}{\rho^{2}} \frac{\partial^{2}}{\partial \varphi^{2}}\right)+\frac{\hbar^{2} q_{z}^{2}}{2 m^{*}}\left(1+\eta_{1}^{2}+\eta_{2}^{2}-2 \eta_{1}-2 \eta_{2}\right) \\
& -\frac{e^{2}}{\varepsilon_{\infty} \sqrt{\left(\rho-\rho_{0}\right)^{2}+z^{2}}}+V_{\mathrm{c}}(r)+V_{\mathrm{LO}}+V_{\mathrm{SO}}+V_{\text {ion-LO }}+V_{\text {ion-SO }} \\
& +V_{\mathrm{e}-\mathrm{LO}-\mathrm{ion}}^{\mathrm{ex}}+V_{\mathrm{e}-\mathrm{SO}-\mathrm{ion} .}^{\mathrm{ex}} .
\end{aligned}
$$

$V_{\mathrm{LO}}$ and $V_{\mathrm{SO}}$ are the effective potentials induced by the interaction between the electron and the confined LO phonon and SO phonon, respectively

$$
V_{\mathrm{LO}}=-\sum_{n, k} \frac{V_{0 n}^{2} J_{0}^{2}\left(\chi_{0 n} \rho / R\right)}{\hbar \omega_{\mathrm{LO}}+\hbar^{2} k^{2} / 2 m^{*}}
$$




$$
V_{\mathrm{SO}}=-\sum_{k} \frac{\Gamma_{0}^{2} I_{0}^{2}(k \rho)}{\hbar \omega_{\mathrm{SO}}+\hbar^{2} k^{2} / 2 m^{*}} .
$$

The effective potentials induced by the ion-phonon (confined LO and SO) coupling are expressed as

$$
\begin{aligned}
& V_{\text {ion-LO }}=-\sum_{n, k} \frac{V_{0 n}^{2} J_{0}^{2}\left(\chi_{0 n} \rho_{0} / R\right)}{\hbar \omega_{\mathrm{LO}}+\hbar^{2} k^{2} / 2 m^{*}}, \\
& V_{\text {ion-SO }}=-\sum_{k} \frac{\Gamma_{0}^{2} I_{0}^{2}\left(k \rho_{0}\right)}{\hbar \omega_{\text {SO }}+\hbar^{2} k^{2} / 2 m^{*}} .
\end{aligned}
$$

We define $V_{\mathrm{e}-\mathrm{LO}-\text { ion }}^{\mathrm{ex}}$ and $V_{\mathrm{e}-\mathrm{ex}}^{\mathrm{ex}}$-ion as the exchange potentials of electron-ion interaction via phonons $\mathrm{LO}$ and $\mathrm{SO}$, respectively

$$
\begin{aligned}
& V_{\mathrm{e}-\mathrm{LO}-\mathrm{ion}}^{\mathrm{ex}}=2 \sum_{n, k} \frac{V_{0 n}^{2} J_{0}\left(\chi_{0 n} \rho / R\right) J_{0}\left(\chi_{0 n} \rho_{0} / R\right) \cos \left[k\left(z_{\mathrm{e}}-z_{0}\right)\right]}{\hbar \omega_{\mathrm{LO}}+\hbar^{2} k^{2} / 2 m^{*}}, \\
& V_{\mathrm{e}-\mathrm{SO}-\text { ion }}^{\mathrm{ex}}=2 \sum_{k} \frac{\Gamma_{0}^{2} I_{0}(k \rho) I_{0}\left(k \rho_{0}\right) \cos \left[k\left(z_{\mathrm{e}}-z_{0}\right)\right]}{\hbar \omega_{\mathrm{SO}}+\hbar^{2} k^{2} / 2 m^{*}}
\end{aligned}
$$

In order to calculate the impurity binding energy, we consider the following ground state of the free electron:

$$
\phi_{\text {free }}=\frac{1}{\sqrt{\pi L_{z}} R J_{1}\left(\chi_{01}\right)} J_{0}\left(\chi_{01} \varrho / R\right) \mathrm{e}^{\mathrm{i} q_{z} z_{\mathrm{e}}} .
$$

The ground state wave function with the impurity is taken as

$$
\phi_{\text {imp }}=N J_{0}\left(\chi_{01} \varrho / R\right) \mathrm{e}^{-\lambda \sqrt{\left(\rho-\rho_{0}\right)^{2}+z^{2}}},
$$

where $N$ is a normalization constant, $z$ coordinate is the relative separation of the electron from the impurity ion along the axis of the QWW and $\lambda$ is a variational parameter, which is determined by minimizing the energy,

$$
N^{-2}=-2 \pi \frac{\mathrm{d} A}{\mathrm{~d} \lambda}
$$

with

$$
A=\int_{0}^{R} \rho J_{0}^{2}\left(\chi_{01} \rho\right) K_{0}\left(2 \lambda \rho_{>}\right) I_{0}\left(2 \lambda \rho_{<}\right) \mathrm{d} \rho,
$$

where $\rho_{>}\left(\rho_{<}\right)$is the greatest (lowest) value of $\rho$ and $\rho_{0}$.

The impurity binding energy is defined as the ground state energy of the system without the Coulomb term, minus the ground state energy in the presence of the Coulomb term,

$$
E_{\mathrm{b}}^{\mathrm{ph}}=\left\langle\phi_{\text {free }}\left|H_{\mathrm{p}}+\frac{e^{2}}{\varepsilon_{\infty} \sqrt{\left(\rho-\rho_{0}\right)^{2}+z^{2}}}\right| \phi_{\text {free }}\right\rangle-\min _{\lambda}\left\langle\phi_{\text {imp }}\left|H_{\mathrm{p}}\right| \phi_{\mathrm{imp}}\right\rangle .
$$

Following Li et al. [35], we can set from Eq. (30):

$$
\left(m_{\mathrm{p}}\right)^{-1}=\left(m_{0}^{*}\right)^{-1}\left(1+\eta_{1}^{2}+\eta_{2}^{2}-2 \eta_{1}-2 \eta_{2}\right) .
$$


The bound polaron's effective mass $m_{\mathrm{p}}^{*}$ can be approximately evaluated by considering $\alpha C \ll 1$ and $\alpha D \ll 1$, as

$$
m_{\mathrm{p}}^{*}=m^{*}(1+2 \alpha C+2 \alpha D)
$$

where $C$ and $D$ are respectively given by Eqs. (27) and (28).

The expected value of $m_{\mathrm{p}}{ }^{*}$ referring to the electronic wave function can be written as

$$
\bar{m}_{\mathrm{p}}^{*}=m^{*}\left\langle\phi_{\mathrm{e}}(\rho, z)|1+2 \alpha C+2 \alpha D| \phi_{\mathrm{e}}(\rho, z)\right\rangle .
$$

\section{Results and discussion}

This section is devoted to the numerical results obtained in the case of CdTe cylindrical quantum well wire embedded in dielectric matrix of constant $\varepsilon_{\mathrm{d}}=1$. The physical parameters corresponding to the polar crystal CdTe are: $\varepsilon_{0}=9.6$, $\varepsilon_{\infty}=7.13, m_{0}^{*}=0.091 m_{0}, \hbar \omega_{\mathrm{LO}}=20.84 \mathrm{meV}, \alpha=0.315, a^{*} \approx 55.91 \AA$ and $R^{*} \approx 13.34 \mathrm{meV}$ where $R^{*}=m^{*} e^{4} / 2 \hbar^{2} \varepsilon_{0}^{2}$ and $a^{*}=\hbar^{2} \varepsilon_{0} / m^{*} e^{2}$ are the effective units of energy and length respectively.

In Fig. 1, the impurity binding energy is plotted against the cylindrical QWW radius $R$ for different impurity positions ( $\rho_{0}=0, \rho_{0}=0.5 R$ and $\rho_{0}=R$ ). The



Fig. 1. Binding energy of a donor impurity as a function of the cylindrical quantum well wires radius for different impurity positions $\left(\rho_{0}=0, \rho_{0}=0.5 R, \rho_{0}=R\right)$. The solid and the dotted curves represent the binding energies without $\left(E_{\mathrm{b}}^{0}\right)$ and with $\left(E_{\mathrm{b}}^{\text {ph }}\right)$ the phonon corrections, respectively. 
figure shows on one hand that the impurity binding energy depends strongly on the size of the wire; when the wire radius decreases, the binding energy, in the presence and absence of the polaronic effect, increases. This is due to the wave function which is more compressed when the wire size decreases, consequently the binding energy enhances. On the other hand, the binding energy depends on the impurity position; as the impurity moves from the center to the edge of the wire, the impurity binding energy diminishes. This result is consistent with that obtained by Brown and Spector [19] in the case when the phonon effect is ignored. The inclusion of the optical phonon leads to an enhancement of the binding energy.

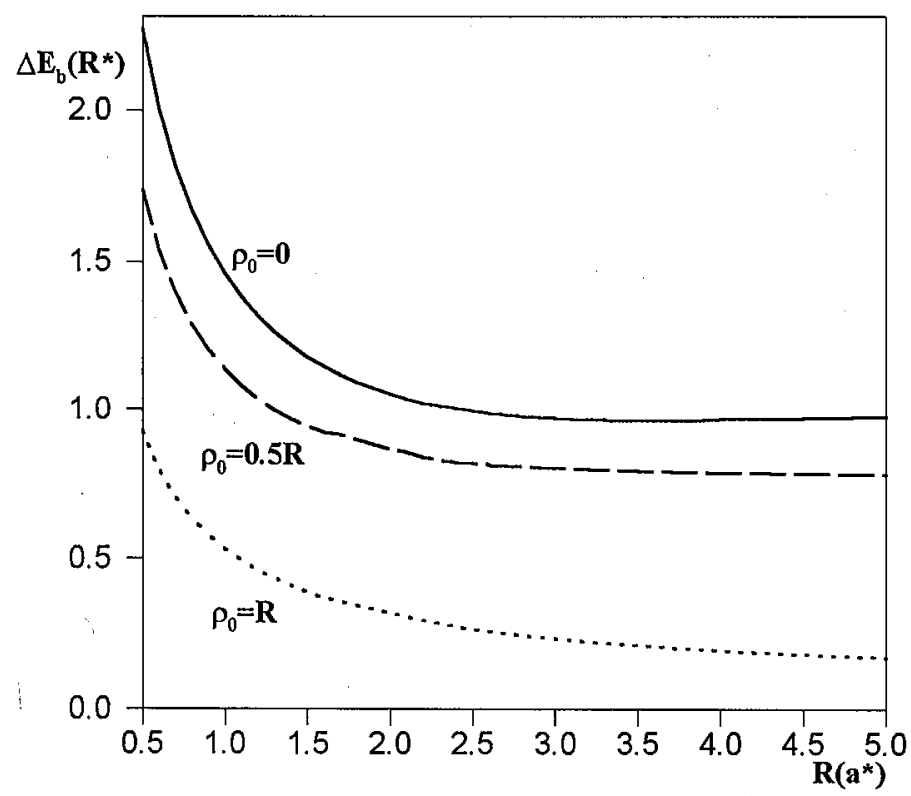

Fig. 2. Shift binding energy $\Delta E_{\mathrm{b}}$ of a donor impurity, due to the polaronic effect plotted versus the wire radius $R$ for different impurity positions ( $\rho_{0}=0, \rho_{0}=0.5 R, \rho_{0}=R$ ).

In order to give a clear picture of the polaronic effect on the donor impurity binding energy, we define the shift energy due to all phonons modes (confined $\mathrm{LO}$ and $\mathrm{SO}$ ) as the difference between the binding energy in the presence and absence of these optical modes $\Delta E_{\mathrm{b}}=E_{\mathrm{b}}^{\mathrm{ph}}-E_{\mathrm{b}}^{0}$. Figure 2 displays the polaronic correction versus the wire radius $R$ for different impurity positions $\left(\rho_{0}=0\right.$, $\rho_{0}=0.5 R$ and $\rho_{0}=R$ ). The shift energy is more pronounced for a strong confinement size and decreases as the QWW radius increases. For an impurity placed at the center of the wire the polaronic corrections are more significant and as the impurity moves away from the center the polaronic corrections become smaller.

To show the dependencies of the impurity binding energy on the impurity position and the polaronic effect, we have plotted in Fig. 3 the binding energy versus the impurity position for different wire radii $\left(R=0.8 a^{*}, R=1 a^{*}\right.$ and $\left.R=1.5 a^{*}\right)$. We can note that for a fixed QWW radius, the binding energies are 


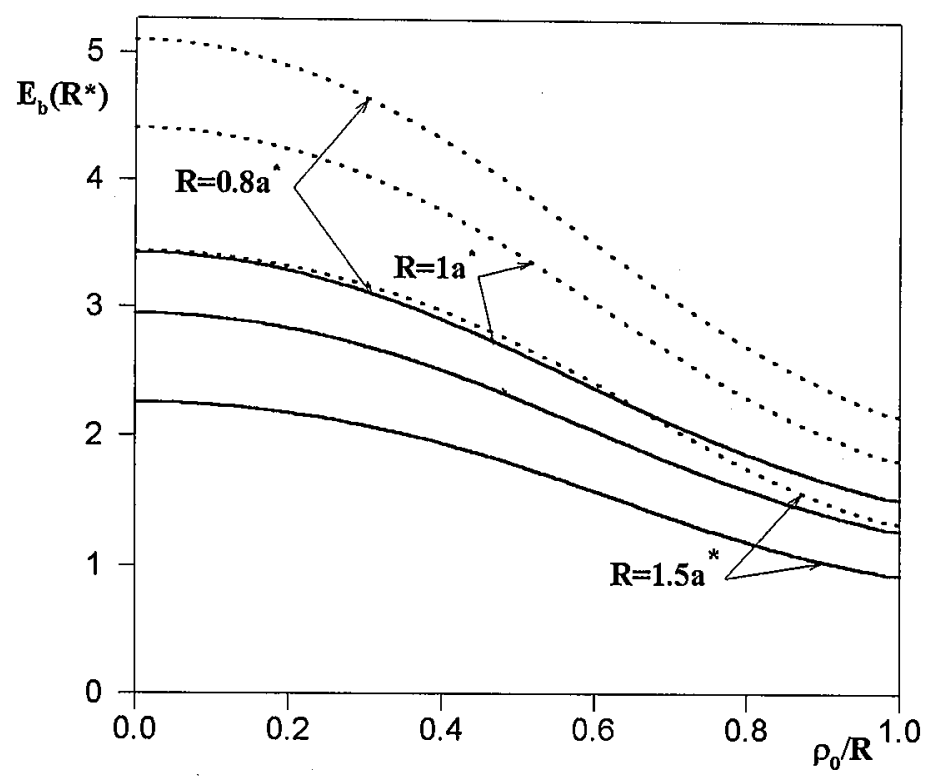

Fig. 3. Binding energy of a donor impurity as a function of the impurity position for different wire radii $\left(R=0.8 a^{*}, R=1 a^{*}\right.$ and $\left.R=1.5 a^{*}\right)$. The solid and dotted curves represent the binding energies without $\left(E_{\mathrm{b}}^{0}\right)$ and with $\left(E_{\mathrm{b}}^{\mathrm{ph}}\right)$ the effect of phonons, respectively.

higher for an on-center impurity and as the impurity moves from the center to the edge of the QWW, the impurity binding energy decreases. This behavior is due to the wave function, which vanishes at the boundaries, and thus its contribution to the binding energy of a QWW with an off-center impurity is smaller than a QWW with an on-center impurity. These results are consistent to that obtained by Montenegro [17] in cylindrical quantum well wire and Bose [16] in spherical quantum dot in the absence of phonons. Figure 4 shows the polaronic correction plotted versus the impurity position for different wire radii $\left(R=0.8 a^{*}, R=1 a^{*}\right.$ and $R=1.5 a^{*}$ ). This shift energy is more significant for an impurity placed at the center and as the impurity moves away from the center, these polaronic corrections become smaller. Furthermore, these corrections are important for a thin wire and decrease as well as the confinement size decreases. An inclusion of the ion-phonons coupling imposes an exchange term (ion-electron interaction via phonons) whose effect is substantial [28]. The contribution of each of the phonon modes to the binding energy has two components: one attractive due to electron-phonon interaction and one repulsive due to the ion-electron interaction via phonon (exchange term). This result is in agreement with that obtained in the case of exciton for LO and SO phonon in Refs. [28] and [29] respectively. The correction of each mode of phonon to the binding energy depends strongly on the behavior of these two components in both ground and subband states. We notice that the optical surface phonons effect is less important than that of confined LO phonon. This result is reflected in Fig. 5, where we have displayed the correction to the effective mass due 


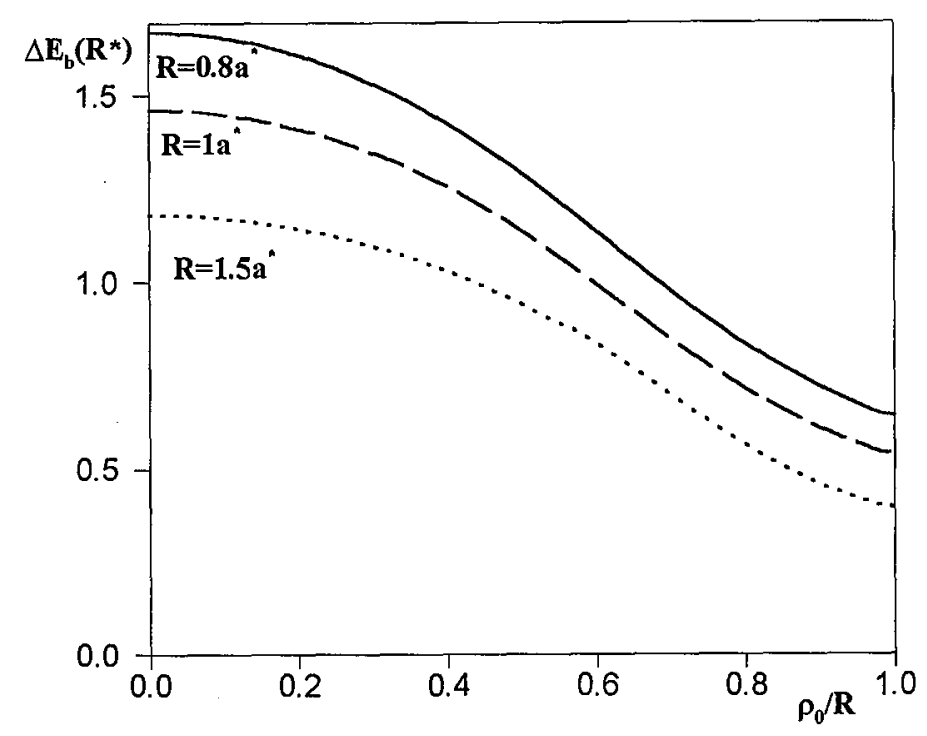

Fig. 4. Shift binding energy plotted versus the impurity position for different cylindrical QWW radii $\left(R=0.8 a^{*}, R=1 a^{*}\right.$, and $\left.R=1.5 a^{*}\right)$.

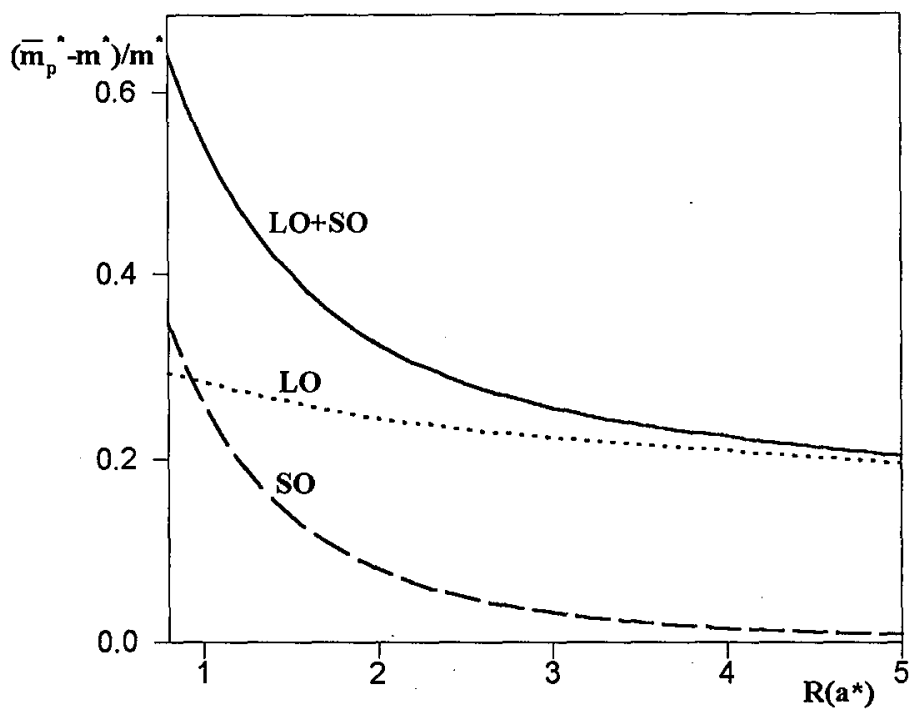

Fig. 5. Corrections of the electron effective mass given by the confined LO and SO phonons and their sum as a function of the QWW radius.

to different phonon modes as a function of the wire radius. We find that the correction due to the SO phonon decreases more rapidly as the wire becomes thicker. The effect of SO phonon is important only for a small wires while the LO phonon contribution is predominant. 


\section{Conclusion}

In conclusion, we have studied the effect of the charge carrier (electron and ion)-phonons (confined LO and SO) coupling on an off-center hydrogenic impurity binding energy in a cylindrical quantum well wire. Our calculations are performed within the effective mass approximation and using the variational treatment. We have considered that the bound electron is confined in an infinitely deep potential well. The results show that polaronic effect is important and cannot be neglected. Furthermore, these corrections as it is shown in the figures depend on the confinement size and the impurity position. The SO phonons are important when the wire size is sufficiently small while the confined LO phonons are predominant when the wire size becomes large.

\section{Acknowledgment}

This work was supported by the Programs-in-Aide for Scientific Research (PARS). Physique 16 Oujda and Physique 03 Fès.

\section{References}

[1] G. Bastard, Phys. Rev. B 24, 4714 (1981).

[2] J. Lee, H.N. Spector, J. Appl. Phys. 57, 366 (1985).

[3] J.W. Brown, H.N. Spector, Phys. Rev. B 35, 3009 (1987).

[4] Z. Xiao, J. Zhu, F. He, Phys. Status Solidi B 191, 401 (1995).

[5] C.A. Duque; A.L. Morales, A. Montes, N.P. Montenegro, Phys. Rev. B 55, 10721 (1997).

[6] C. Bose, J. Appl. Phys. 83, 3089 (1997).

[7] M.H. Degani, O. Hipolito, Phys. Rev. B 35, 4507 (1987).

[8] H.J. Xie, C.Y. Chen, S.D. Liang, Phys. Rev. B 52, 1776 (1995).

[9] H.J. Xie, C.Y. Chen, Y.Y. Liu, Physica B 253, 73 (1998).

[10] V.V. Pranjape, Phys. Rev. B 53, 6908 (1996).

[11] P. Villamil, N. Porras-Montenegro, J.C. Granada, Phys. Rev. B 59, 1605 (1999).

[12] P. Villamil, N. Porras-Montenegro, J. Phys., Condens. Matter 10, 10599 (1998).

[13] A. Kumar, S.E. Laux, F. Stern, Phys. Rev. B 42, 1566 (1990).

[14] F.J. Ribeiro, A. Latgé, M. Pacheco, Z. Barticevic, J. Appl. Phys. 82, 270 (1997).

[15] S. Mukhopadhyay, A. Chattertjee, Phys. Rev. B 55, 5944 (1997).

[16] C. Bose, C.K. Sarkar, Physica B 253, 238 (1998).

[17] N. Porras-Montenegro, J. Lopez-Gondar, L.E. Oliveira, Phys. Rev. B 43, 1824 (1991).

[18] S.V. Branis, Gang Li, K.K. Bajaj, Phys. Rev. B 47, 1316 (1993).

[19] J.W. Brown, H.N. Spector, J. Appl. Phys. 59, 1179 (1986).

[20] A. Montes, C.A. Duque, N.P. Montenegro, J. Appl. Phys. 81, 7890 (1997).

[21] Z. Xiao, J. Zhu, F. He, J. Appl. Phys. 79, 9181 (1996).

[22] A. Montes, C.A. Duque, N.P. Montenegro, Phys. Status Solidi B 210, 731 (1998). 
[23] J.T. Devreese, in: Encyclopedia of Applied Physics, Vol. 14, VCH Publishers, Weinheim 1996, p. 383.

[24] H.Y. Zhou, S.W. Gu, Solid State Commun. 88, 291 (1993).

[25] W.D. Sheng, Y.Q. Xiao, S.W. Gu, J. Phys., Condens. Matter 5, L129 (1993).

[26] R. Zheng, M. Matsuura, Phys. Rev. B 56, 2058 (1997).

[27] C.Y. Chen, P.W. Jin, W.S. Li, D.L. Lin, Phys. Rev. B 56, 14913 (1997).

[28] J.C. Marini, B. Stebe, E. Khartheuser, Phys. Rev. B 50, 14302 (1994).

[29] W.D. Sheng, S.W. Gu, Solid State Commun. 88, 111 (1993).

[30] S.N. Klimin, E.P. Pokatilov, V.M. Fomin, Phys. Status Solidi B 184, 373 (1994).

[31] X.F. Wang, X.L. Lei, Phys. Rev. B 49, 4780 (1994).

[32] T.D. Lee, F. Low, D. Pines, Phys. Rev. 90, 297 (1953).

[33] N.C. Constantinou, B.K. Ridley, Phys. Rev. B' 41, 10622 (1990).

[34] N.C. Constantinou, B.K. Ridley, J. Phys., Condens. Matter 1, 2283 (1989).

[35] Y.C. Li, L.F. Zheng, S.W. Gu, Phys. Rev. B 38, 4096 (1988). 